\title{
Non-invasive remote limb ischemic postconditioning protects rats against focal cerebral ischemia by upregulating STAT3 and reducing apoptosis
}

\author{
ZHIGANG CHENG, LING LI, XUEYING MO, LU ZHANG, YONGQIU XIE, QULIAN GUO and YUNJIAO WANG \\ Department of Anesthesiology, Xiang Ya Hospital, Central South University, Changsha, Hunan 410008, P.R. China
}

Received March 22, 2014; Accepted July 24, 2014

DOI: $10.3892 / \mathrm{ijmm} .2014 .1873$

\begin{abstract}
The signal transducer and activator of transcription 3 (STAT3) signaling pathway has been implicated in cell apoptosis and inflammatory processes. Ischemic preconditioning (IPC) and ischemic postconditioning (IPTC) inhibit both of these processes. In the present study, we investigated the role of phosphorylated STAT3 (p-STAT3)-mediated apoptosis and inflammation following non-invasive remote limb IPTC (NRIPoC) using a classic rat model of focal cerebral ischemia. Forty-five adult male Sprague-Dawley rats were divided randomly into 3 groups ( $n=15$ per group): the shamoperated, ischemia/reperfusion (I/R) and NRIPoC groups. NRIPoC was implemented at the beginning of reperfusion. At $24 \mathrm{~h}$ after cerebral reperfusion, we evaluated the neurological deficit score (NDS), assessed the cerebral infarct size and tissue morphology, and evaluated neuronal apoptosis. The protein expression levels of Bcl-2, Bax, nuclear factor- $\kappa \mathrm{B}$ $(\mathrm{NF}-\kappa \mathrm{B})$, tumor necrosis factor- $\alpha$ (TNF- $\alpha)$ and p-STAT3 in the penumbra region were assessed by western blot analysis. The cerebral infarct volume, the number of apoptotic cells and the protein expression levels of Bcl-2, Bax, NF- $\kappa$ B and TNF- $\alpha$ were all found to be increased in the I/R group compared with the sham-operated group. However, these levels were decreased in the NRIPoC group compared with the I/R group. The number of apoptotic cells in the penumbra in the I/R group was increased compared with that in the NRIPoC and sham-operated groups. The protein expression of p-STAT3 was increased in the NRIPoC group compared with the sham-operated and I/R groups. These results indicate that the protective effects of NRIPoC against cerebral I/R injury may
\end{abstract}

Correspondence to: Professor Yunjiao Wang, Department of Anesthesiology, Xiang Ya Hospital, Central South University, 87 Xiang Ya Road, Changsha, Hunan 410008, P.R. China

E-mail: yunjiaowang@hotmail.com

Key words: ischemic postconditioning, cerebral ischemia, apoptosis, inflammation be related to the attenuation of neuronal apoptosis and inflammation through the activation of STAT3.

\section{Introduction}

Ischemic stroke is a major cause of death and disability worldwide, and the clinical prognosis of acute cerebral ischemia is poor $(1,2)$. The early reperfusion after cerebral ischemia is essential for the viability and functional recovery of the brain; however, the arrival of blood oxygen to the ischemic tissue causes ischemia/reperfusion injury (IRI), which induces further damage (3). Since 1986, when Murray et al (4) first described a classic phenomenon they termed ischemic preconditioning (IPC), it has been repeatedly confirmed in animal models that IPC and ischemic postconditioning (IPTC) are powerful endogenous protective strategies against IRI of multiple organs, including the heart, brain and kidneys (8). However, the clinical implementation of IPC and IPTC as a means of protection against IRI is not desirable or feasible in most clinical circumstances due to the unpredictability of organ ischemia and the possibility that added ischemia may result in dangerous complications (9). The application of brief episodes of ischemia to a tissue remote from the primary ischemic organ (e.g., heart or brain) using the techniques of remote ischemic preconditioning (RIP) or remote ischemic postconditioning (RIPoC) may confer cerebral/cardiac protection, and RIPoC can overcome the aforementioned issues previously associated with IPC and IPTC (10-17). Both RIP and $\mathrm{RIPoC}$ have been recognized as applicable strategies for the protection against cerebral and myocardial IRI $(11,13,18-22)$. A further refinement of RIPoC, non-invasive remote ischemic postconditioning (NRIPoC), can be applied in a wider range of clinical settings for cerebral and myocardial IRI due to its practicality as a non-invasive technique. NRIPoC is a relatively recent innovation $(11,23-25)$, and its fundamental biology is not yet well understood.

Apoptosis and the inflammatory response both play fundamental pathogenic roles in cerebral IRI (26-32). Tumor necrosis factor- $\alpha$ (TNF- $\alpha)$ is the most important pro-inflammatory cytokine involved in cerebral IRI, and its effects are observed throughout the development of inflammation (33-35). Nuclear factor $-\kappa \mathrm{B}(\mathrm{NF}-\kappa \mathrm{B})$ regulates the gene expression of inflammatory factors involved in the ischemia/reperfusion $(\mathrm{I} / \mathrm{R})$ of 
the cerebral tissue $(36,37)$. The inflammatory response can induce apoptosis by regulating apoptotic signals during cerebral IRI (38-41). Evidence indicates that the Janus kinase (JAK)/ signal transducer and activator of transcription 3 (STAT3) signaling pathway transduces stress-activating extracellular chemical signals into cellular responses for a number of pathophysiological processes, such as immunity, inflammation and apoptosis, and is involved in cerebral IRI (42-45). We hypothesized that the NRIPoC-induced neuroprotective effects may be associated with the activation of the STAT3 signaling pathway, the inhibition of the inflammatory response and the regulation of apoptosis. To explore this hypothesis, we performed experiments to evaluate the roles of apoptosis, the inflammatory response and STAT3 signaling in cerebral protection conferred by NRIPoC during focal cerebral IRI in an in vivo rat model.

\section{Materials and methods}

Animals. Male Sprague-Dawley rats weighting 250-300 g were purchased from the Center of Laboratory Animals of Central South University, Changsha, China. The rats were placed in a room with a controlled environment with a 12-/12-h light/dark cycle and allowed access ad libitum to standard rodent chow and tap water. This study was approved by the Institutional Animals Ethics Committee of Central South University and was conducted in accordance with the Guidelines for the Care and Use of Laboratory Animals provided by the National Institutes of Health (NIH publication no. 80-82).

Model of focal cerebral ischemia. Middle cerebral artery (MCA) occlusion (MCAO) was carried out as previously described (46). The animals were anesthetized by an intraperitoneal injection of $300 \mathrm{mg} / \mathrm{kg}$ chloral hydrate. Heating lamps were used to maintain rectal temperature at $37-37.5^{\circ} \mathrm{C}$. The right common carotid artery (CCA), external carotid artery (ECA) and internal carotid artery (ICA) were exposed through a midline neck incision, and the ECA was ligated close to its origin with a 3-0 silk suture. A 0.26-mm monofilament nylon suture with a blunt tip (Beijing Shandong Industrial Corp., Beijing, China) was inserted into the ICA, and advanced 18-20 mm until mild resistance was felt, effectively occluding the MCA. After 90 min of MCAO, the monofilament nylon suture was removed and ICA perfusion was restored.

Experimental protocol. In total, 45 rats were randomly assigned to 3 groups ( $\mathrm{n}=15$ in each group): the sham-operated, I/R and NRIPoC groups (Fig. 1). The I/R group underwent MCAO by occlusion of the right MCA for $90 \mathrm{~min}$, followed by $24 \mathrm{~h}$ of cerebral reperfusion. The sham-operated group underwent the same procedure as the I/R rats, but without occlusion of the right MCA. The NRIPoC group underwent the same procedure as the I/R group, but was also subjected to sequential I/R, which involved 3 applications of ischemia for $5 \mathrm{~min}$ and reperfusion for $5 \mathrm{~min}$ to the right hind limb during MCA recovery reperfusion.

To carry out this procedure, a modified non-invasive blood pressure cuff was strapped to the base of the right hind limb of the rat, and pulse sensors were placed on the area of the dorsalis pedis artery. NRIPoC was induced by increasing the pressure of the cuff until blood flow in the limb was blocked. This was

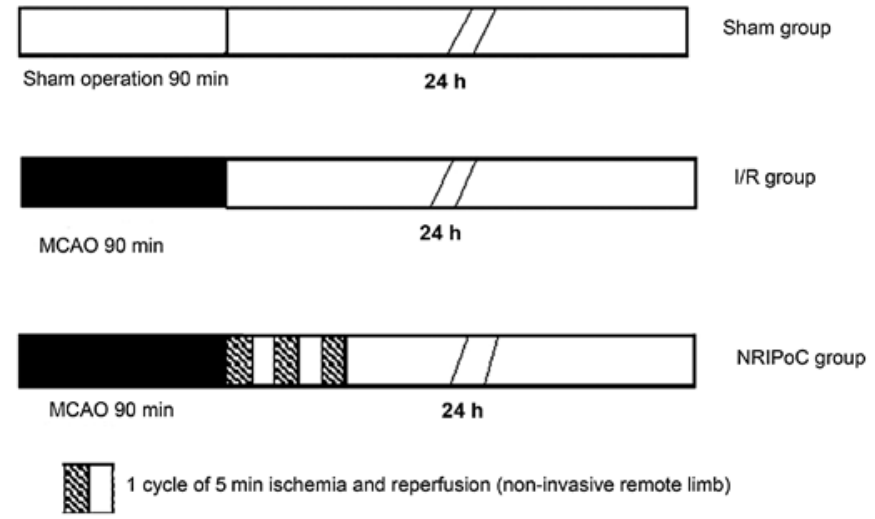

Figure 1. Illustration of the experimental protocol. The sham-operated (sham) group underwent the operation without right middle cerebral artery (MCA) occlusion. Both the ischemia/reperfusion (I/R) and non-invasive remote ischemic postconditioning (NRIPoC) groups underwent MCA occlusion (MCAO) by occlusion of the right MCA for $90 \mathrm{~min}$, followed by $24 \mathrm{~h}$ of reperfusion. In addition, the NRIPoC group underwent sequential I/R of the right hind limb, using 3 cycles of $5 \mathrm{~min}$ of ischemia and $5 \mathrm{~min}$ of reperfusion following MCA reperfusion. Five rats were randomly selected from each group to assess the cerebral infarction volume [histological and 2,3,5-triphenyltetrazolium chloride (TTC) staining] and for western blot analyses.

maintained for $5 \mathrm{~min}$ and then released for $5 \mathrm{~min}$, beginning the onset of MCA reperfusion. This was carried out 3 times for 10-min cycles. The blood flow of the right hind limb was completely blocked by the cuff as confirmed by the disappearance of the pulse and hypothermia and skin cyanosis in the limb.

Neurological evaluation. The animals were returned to their cages after the procedures were finished and again allowed free access to food and water. The neurological deficit score (NDS), as previously described by Longa et al (46), was measured to assess neurological evaluation at $24 \mathrm{~h}$ after reperfusion as follows: 0 , no deficit; 1 , failure to extend left forepaw fully; 2, circling to the left; 3 , falling to the left; and 4, no spontaneous walking with a depressed level of consciousness.

Measurement of infarct volume. After a reperfusion period of $24 \mathrm{~h}$, the cerebral infarct area was identified by 2,3,5-triphenyltetrazolium chloride (TTC) staining as previously described $(47,48)$. The infarct volume was calculated according to the following formula: $\mathrm{V}=\mathrm{t} \times(\mathrm{A} 1+\mathrm{A} 2 \ldots+\mathrm{An})$, where ' $\mathrm{t}$ ' is the brain slice thickness and ' $\mathrm{A}$ ' is the infarct area. The percentage cerebral infarct volume was calculated as follows: (cerebral infarct total volume/whole brain volume) x100.

Preparation of cerebral specimens. After a reperfusion period of $24 \mathrm{~h}, 5$ rats in each group were randomly selected and anesthetized with chloral hydrate. Approximately 300-400 $\mathrm{ml}$ normal saline was infused via an aortic root catheter until the liver appeared white, followed by $200 \mathrm{ml}$ $4 \%$ paraformaldehyde solution that had been cooled to $4^{\circ} \mathrm{C}$. The brain was removed, fixed in $4 \%$ paraformaldehyde solution for $24 \mathrm{~h}$, and 4-mm-thick coronal sections were then cut from the front of the brain through the optic chiasm. After gradient ethanol dehydration, vitrification with isobutanol and n-butyl alcohol and embedding in paraffin wax, the slices 
were cut into 5- $\mu$ m-thick coronal sections on a vibratome, and the sections were dried overnight in an incubator at $37^{\circ} \mathrm{C}$ for later processing with hematoxylin and eosin (H\&E) and Nissl staining, terminal deoxynucleotidyl transferase-mediated dUTP nick end-labelling (TUNEL).

Assessment of neuronal survival. The coronal brain sections were examined using a standard hematoxylin and eosin (H\&E) staining protocol to examine cellular morphology by observing the number and shape of the neurons. The brain sections were stained with toluidine blue for Nissl bodies. Neuronal survival was assessed by observing the Nissl bodies in the neurons. Images of the ischemic penumbras of the 5 rats were captured, and quantitative analyses of the cells were performed using Image-Pro Plus 6.0 software (Media Cybernetics, Inc., Rockville, MD, USA).

Assessment of apoptosis. Apoptosis was measured by TUNEL assay using an Apoptosis Detection kit (Roche Applied Science, Mannheim, Germany), in accordance with the manufacturer's instructions. TUNEL-positive neurons were viewed under a light microscope (Leica DM 2000; Leica Microsystems, Wetzlar, Germany) and were identified by bluish-violet-stained nuclei. Five visual fields were randomly selected from the ischemic penumbra of each slice at $\mathrm{x} 400$ magnification. The apoptotic cells were counted in each field, and the average value of the 5 fields was calculated.

Western blot analysis. After a reperfusion period of $24 \mathrm{~h}$, the brains of the 5 rats in each group selected randomly were rapidly removed under deep anesthesia, and the right ischemic penumbra areas of the parietal cortex were immediately isolated onto ice held at $-20^{\circ} \mathrm{C}$. The pulverized brain samples weighing $0.25 \mathrm{~g}$ were homogenized with $500 \mu \mathrm{l}$ protein lysis buffer. The homogenates were centrifuged at $12,000 \mathrm{rpm}$ for $5 \mathrm{~min}$ at $4^{\circ} \mathrm{C}$, and the supernatants were collected for western blot analysis. The protein concentrations were determined by bicinchoninic acid (BCA) protein assays (WellBiz Brands, Inc., Highlands Ranch, CO, USA). Western blot analyses were carried out using standard techniques (49). Briefly, a mixture of homogenate sample containing $30 \mu \mathrm{g}$ protein and $5 \mathrm{X}$ sodium dodecyl sulfate (SDS) loading buffer was boiled at $100^{\circ} \mathrm{C}$ for $5 \mathrm{~min}$, separated on $10 \%$ SDS-polyacrylamide gels, transferred onto polyvinylidene fluoride (PVDF) membranes (Pierce Biotechnology, Inc., Rockford, IL, USA) and blocked for $1 \mathrm{~h}$ with $5 \%$ non-fat powdered milk in $100 \mathrm{mM}$ Tris-buffered saline containing $0.05 \%$ Tween-20 (TBST). The membranes were incubated overnight at $4{ }^{\circ} \mathrm{C}$ with antibodies against Bcl-2 (Abcam plc., Cambridge, UK), Bax (Cell Signaling Technology, Inc., Beverly, MA, USA), phosphorylated STAT3 (p-STAT3), or $\beta$-actin (both Santa Cruz Biotechnology, Inc., Santa Cruz, CA, USA). After 3 washes in TBST, the membranes were incubated with secondary antibody (HRP rabbit anti-goat IgG; Santa Cruz Biotechnology, Inc.) for $1 \mathrm{~h}$ at room temperature, followed by 3 washes in TBST. Signals were detected with an Enhanced Chemiluminescence kit (Pierce Biotechnology, Inc.).

Statistical analysis. Data analyses were performed using SPSS software 13.0 (SPSS Inc., Chicago, IL, USA). NDS values are expressed as the median (range) and were compared

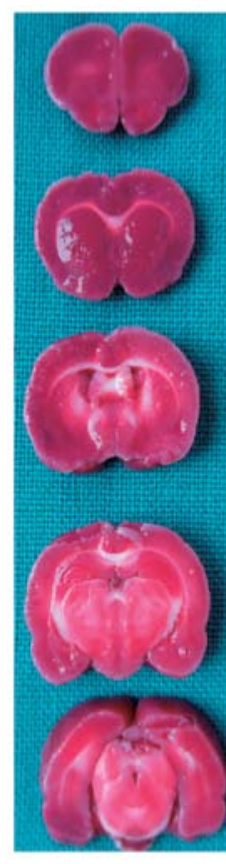

sham

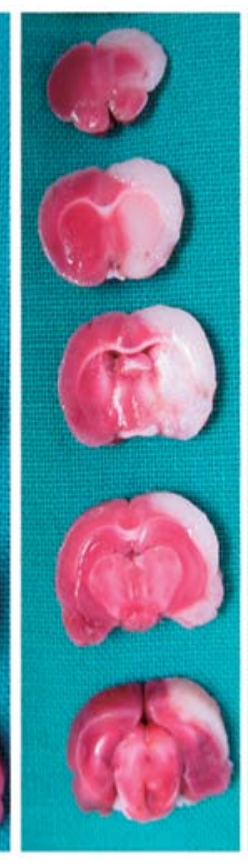

I/R

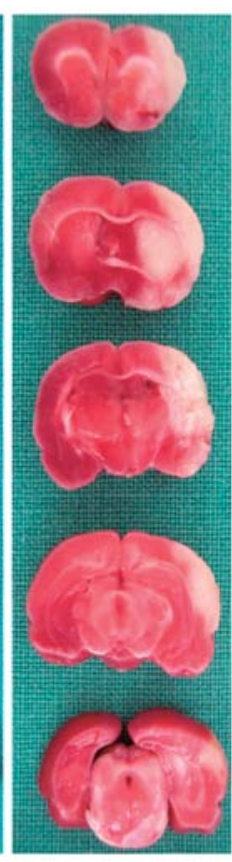

NRIPoC
Figure 2. Effects of non-invasive remote ischemic postconditioning (NRIPoC) on the ischemia reperfusion injury (IRI)-induced cerebral infarction volume determined by 2,3,5-triphenyltetrazolium chloride (TTC) staining $(n=5)$; normal brain tissue is red, infarcted brain tissue is white.

using Kruskal-Wallis tests. Other data are presented as the means \pm standard deviation (SD). One-way analysis of variance (ANOVA) followed by Student's t-tests were applied to determine differences between groups. A value of $\mathrm{P}<0.05$ was considered to indicate a statistically significant difference.

\section{Results}

Effects of NRIPoC on the IRI-induced cerebral infarct volume. The cerebral infarct areas determined by TTC staining are illustrated in Fig. 2. There were no conspicuous cerebral infarcts area in the sham-operated group rats, while the cerebral infarct volumes of the I/R and NRIPoC rats were 28.4 \pm 3.7 and $15.2 \pm 6.9$, respectively. The cerebral infarct volumes were significantly decreased in the NRIPoC group compared with the $\mathrm{I} / \mathrm{R}$ group $(\mathrm{P}<0.05)$.

Effects of NRIPoC on IRI-induced neuronal loss. Nissl bodies were used as a morphological indicator of neuronal survival. The number of Nissl-stained neurons in the ischemic penumbra areas in the I/R and NRIPoC groups was significantly reduced compared with the sham-operated group $(\mathrm{P}<0.05)$; however, this decrease was less significant in the NRIPoC group compared with the marked decrease observed in the $\mathrm{I} / \mathrm{R}$ group (Fig. 3A-C and E). The Nissl bodies in the ischemic core areas in the I/R and NRIPoC groups were wiped out in vast numbers, and there was no significant difference between the 2 groups (Fig. 3D and F).

Effects of NRIPoC on IRI-induced neuronal morphological changes. Neuronal morphology was assessed by H\&E 

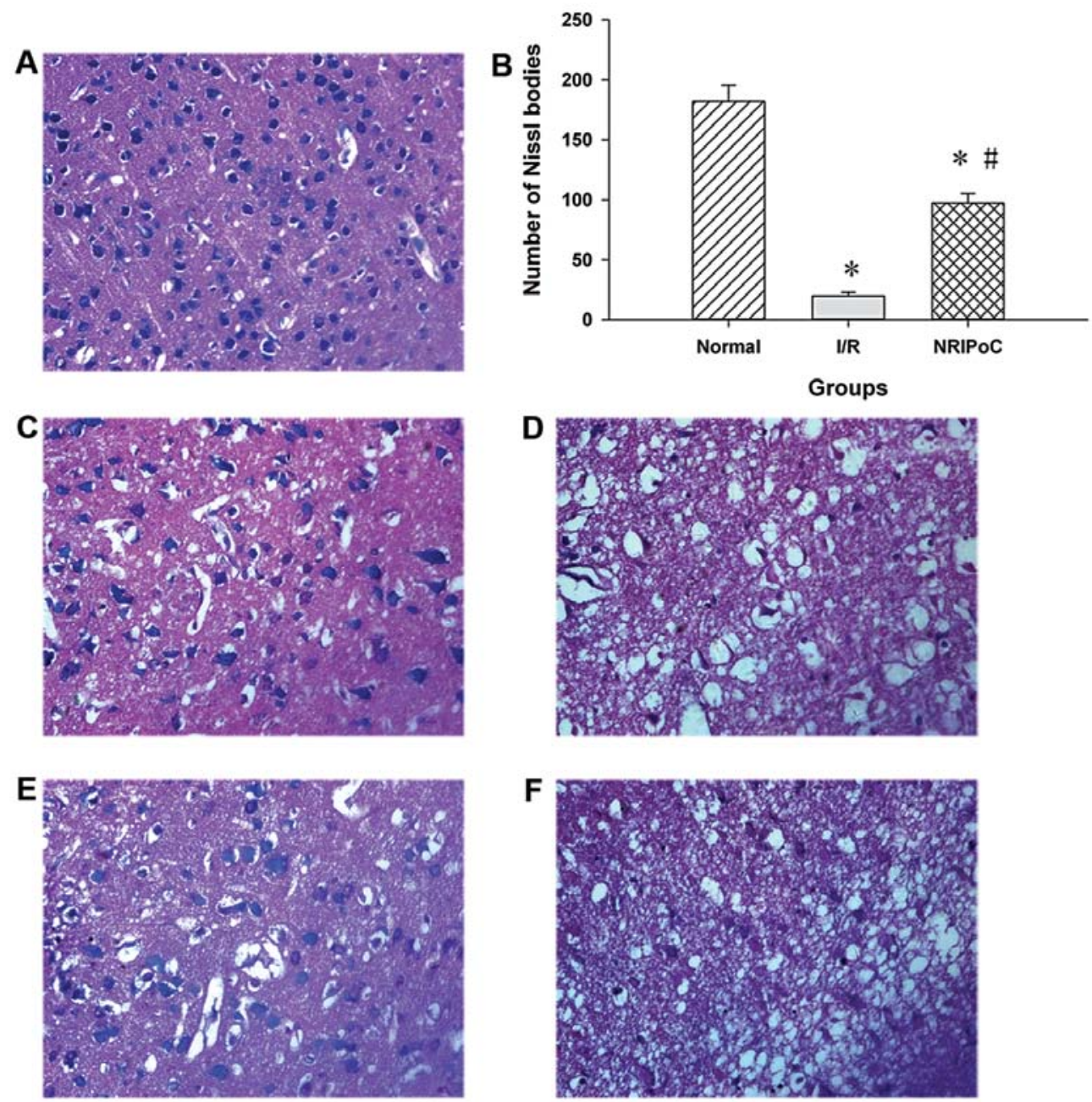

Figure 3. Nissl bodies in the penumbra and core area in the 3 groups at $24 \mathrm{~h}$ after reperfusion $(\mathrm{n}=5)$. Nissl bodies are shown at $\mathrm{x} 400$ magnification. In the rat cortex, Nissl bodies appear as large granular basophilic bodies in the neuronal cytoplasm and are composed of a rough endoplasmic reticulum. The damaged neurons were identified by the loss of Nissl substance. Data are presented as the means \pm standard deviation (SD). ${ }^{*} \mathrm{P}<0.05$ compared with the sham-operated (normal) group; ${ }^{\text {"}} \mathrm{P}<0.05$ compared with the ischemia/reperfusion (I/R) group. (A) Cortex in the sham-operated group; (B) statistical histogram of Nissl bodies in the 3 groups; (C) penumbra cortex in the I/R group; (D) ischemic core in the I/R group; (E) penumbra cortex in the non-invasive remote ischemic postconditioning (NRIPoC) group; (F) ischemic core in the NRIPoC group.

staining (Fig. 4). The number of neurons in the ischemic penumbra area of the $\mathrm{I} / \mathrm{R}$ group was reduced. The obvious characteristics of the neurons were: decreased cell size, nuclear pyknosis, interstitial edema, cell disorder and chromatin condensation. Compared with the I/R group, the NRIPoC group showed a marked improvement in the cellular morphology of the ischemic penumbra area, as only a few neurons were observed to have nuclear pyknosis, hyperchromasis and extremely loose organization. There was no obvious difference in cellular morphology in the ischemic core area between the I/R and NRIPoC groups.

Effects of NRIPoC on IRI-induced neuronal apoptosis. Apoptosis was determined by TUNEL staining (Fig. 5), which revealed that there were few apoptotic neurons in the shamoperated group. In the I/R group, there were large numbers of apoptotic neurons in the ischemic penumbra area; however, NRIPoC significantly decreased these numbers $(P<0.05)$. There were numerous apoptotic neurons in the ischemic core areas of both the I/R and NRIPoC groups.
Effects of NRIPoC on IRI-induced Bcl-2 and Bax expression in the ischemic penumbra area. Bcl-2 and Bax protein levels were significantly increased in the I/R and NRIPoC groups compared with the sham-operated group $(\mathrm{P}<0.05)$ (Fig. 6). NRIPoC significantly increased Bcl-2 protein expression and decreased Bax protein expression following IRI $(\mathrm{P}<0.05)$. There was no difference in the $\mathrm{Bcl}-2 / \mathrm{Bax}$ ratio between the $\mathrm{I} / \mathrm{R}$ and sham-operated groups; however, this ratio was significantly increased in the NRIPoC group compared with the I/R group $(\mathrm{P}<0.05)$.

Effects of NRIPoC on p-STAT3 protein expression in the ischemic penumbra area. The expression of p-STAT3 protein in the ischemic penumbra area was significantly increased following IRI $(\mathrm{P}<0.05)$ (Fig. 7). NRIPoC significantly increased p-STAT3 protein expression in the ischemic penumbra area compared with the $\mathrm{I} / \mathrm{R}$ group $(\mathrm{P}<0.05)$.

Effects of NRIPoC on I/R-induced NF- $\kappa B$ and TNF- $\alpha$ expression in the ischemic penumbra area. The NF- $\mathrm{kB}$ and TNF- $\alpha$ 
Table I. Neurological deficit score of the rats in this study.

\begin{tabular}{lcccccc}
\hline & \multicolumn{4}{c}{ Neurological deficit score } & Median \\
\cline { 2 - 6 } Group & 0 & 1 & 2 & 3 & 4 & (minimum-maximum) \\
\hline Sham & 15 & 0 & 0 & 0 & 0 & 0 \\
I/R & 0 & 0 & 5 & 10 & 0 & $3(2-3)$ \\
NRIPoC & 0 & 3 & 6 & 6 & 0 & $2(1-3)$ \\
\hline
\end{tabular}

Sham, sham-operated; I/R, ischemia/reperfusion; NRIPoC, non-invasive remote ischemic postconditioning.

A
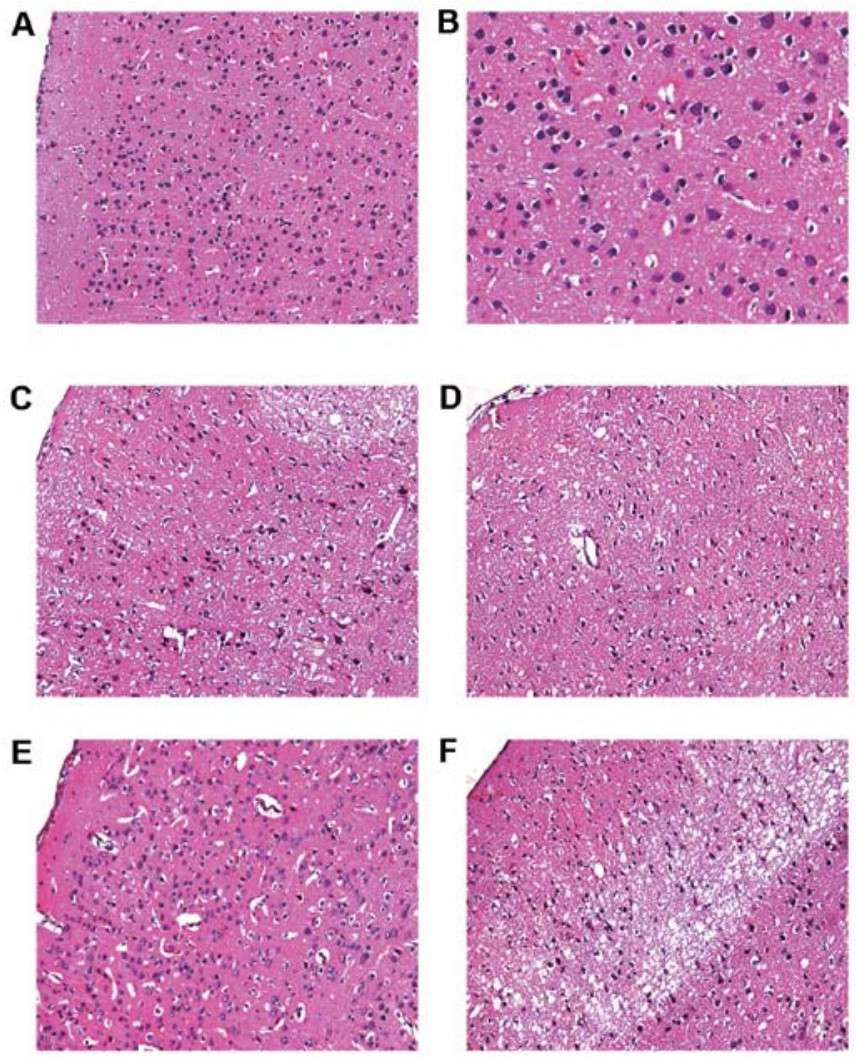

Figure 4. The cortex morphology assessed by hematoxylin and eosin (H\&E) staining in the 3 groups at $24 \mathrm{~h}$ after reperfusion. All images are x200 magnification with the exception of (B) (x400). The neuronal morphology of the sham-operated (sham) group cortex was normal. The cellular morphology of the ischemic penumbra tissue in the non-invasive remote ischemic postconditioning (NRIPoC) group was markedly improved compared with the ischemia/reperfusion (I/R) group. There were a few neurons in the ischemic core areas of the I/R and NRIPoC groups. (A and B) Sham-operated group cortex; (C) ischemic penumbra of the I/R group; (D) ischemic core area of the I/R group; (E) ischemic penumbra of the NRIPoC group; $(\mathrm{F})$ ischemic core area of NRIPoC group.

protein expression levels in the ischemic penumbra area were significantly increased following IRI $(\mathrm{P}<0.05)$ (Fig. 8). NRIPoC significantly decreased NF- $\kappa \mathrm{B}$ and TNF- $\alpha$ expression compared with the I/R group $(\mathrm{P}<0.05)$; however, the levels did not return to those of the sham-operated group $(\mathrm{P}<0.05)$.

Effects of NRIPoC on neurological function. Neurological function in the sham-operated group was normal (NDS $=0$ )
(Table I). The median NDS of the NRIPoC group was 1 score lower on the scale than that in the I/R group, but there was no statistically significant difference between the 2 groups $(\mathrm{P}>0.05)$, and the median NDS in the NRIPoC group was still higher than that of the sham-operated group $(\mathrm{P}<0.01)$.

\section{Discussion}

In this study, we used an in vivo model of MCAO-induced focal ischemia, which has the advantage of being simple and reliable without requiring a craniectomy. MCAO has been widely used to study the effects and mechanisms of pre- and postconditioning on focal cerebral ischemia. Although there have been various ischemic penumbra definitions following focal cerebral ischemia put forward over the 30 years since ischemic penumbra was first introduced by Astrup et al (50) in 1981 , salvaging the ischemic penumbra is the current primary therapeutic target in acute stroke (51). Following cerebral ischemia reperfusion, many neurons in the ischemic penumbra or peri-infarct zone may undergo apoptosis after several hours or days. This renders the time window of therapeutic opportunity for salvaging those cells of the ischemic penumbra.

We hypothesized that the neuroprotective effects of NRIPoC may be mediated through its effects on the ischemic penumbra. The results from the present study demonstrate that NRIPoC significantly increased the number of surviving neurons and decreased neuronal apoptosis in the ischemic penumbra following IRI in a rat model of focal cerebral ischemia induced by 90 min of MCAO. Conversely, there was no difference in neuronal survival in the ischemic core between the I/R and NRIPoC groups. The finding that NRIPoC significantly decreased neuronal apoptosis in the penumbra are is consistent with the results of a previous study demonstrating that RIPoC significantly attenuated neuronal apoptosis associated with global cerebral ischemia in the hippocampal CA1 region (19). TTC is a light-sensitive compound that reacts with lactate dehydrogenase (LDH), which stains normal brain tissue red, while ischemic brain tissue remains white. In the present study, TTC staining revealed that NRIPoC significantly reduced the cerebral infarct size, suggesting that NRIPoC increased cell viability in the cerebral penumbra area. This observation is in accordance with the results of previous studies by Ren et al $(13,17)$.

In addition to necrosis, there is overwhelming evidence suggesting that apoptosis significantly contributes to cell death following cerebral IRI $(27,52,53)$. Preventing apoptosis in the 

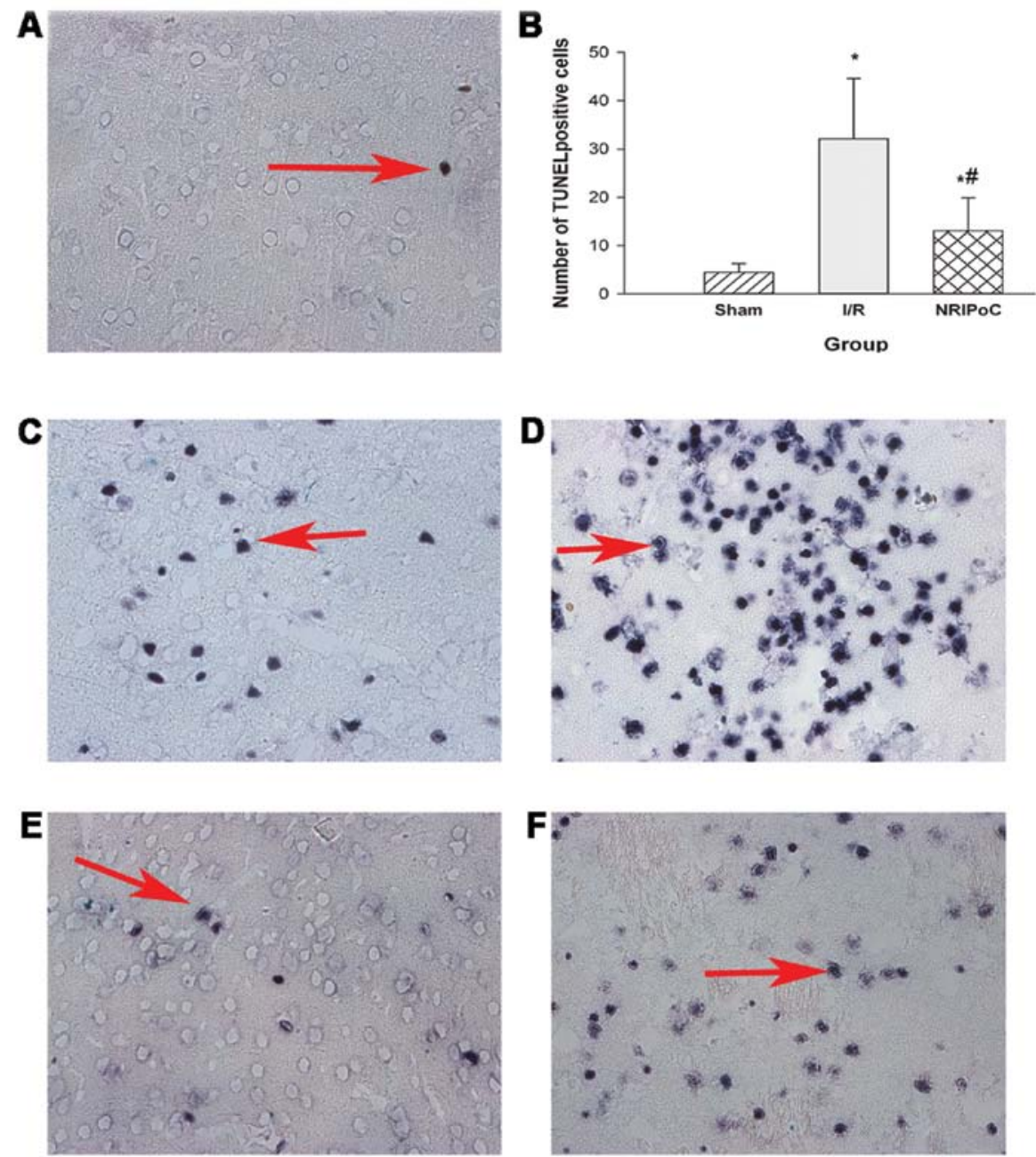

Figure 5. Effects of non-invasive remote ischemic postconditioning (NRIPoC) on ischemia reperfusion injury (IRI)-induced neuronal apoptosis determined by TUNEL staining at $24 \mathrm{~h}$ after reperfusion $(\mathrm{n}=5)$. The TUNEL-positive cells are shown at $\mathrm{x} 400$ magnification. Arrows indicate TUNEL-positive cells. The features of TUNEL-positive cells observed under a light microscope were as follows: violet granules, uneven nuclei, an irregular shape, nuclear pyknosis and nuclear fragmentation. Data are presented as the means \pm standard deviation $(\mathrm{SD}) .{ }^{*} \mathrm{P}<0.05$ compared with the sham-operated (sham) group; ${ }^{*} \mathrm{P}<0.05$ compared with the I/R group. (A) Sham-operated group cortex; (B) I/R group cortex; (C) ischemic penumbra of the I/R group; (D) ischemic core area of the I/R group; (E) ischemic penumbra of the NRIPoC group; $(F)$ ischemic core area of the NRIPoC group.

penumbra area may reduce neuronal loss and limit cerebral IRI. Ischemia pre- and postconditioning has been shown to decrease neuronal apoptosis and attenuate IRI in brain tissue (6,54-57). Similarly, NRIPoC significantly decreased neuronal apoptosis and reduced the cerebral infarct volume after focal cerebral ischemia in the present study. These results suggest that anti-apoptotic signaling may be an important mechanism responsible for the neuroprotective effects of NRIPoC.

Bcl-2 family members are key regulators of apoptosis and modulate mitochondrial membrane permeability. Following cell ischemia or hypoxia, the expression of mitochondrial membrane proteins is increased, and the apoptotic protein, Bax, which translocates from the cytoplasm into the mitochondria, promotes the release of cytochrome $c$, while the anti-apoptotic protein, $\mathrm{Bcl}-2$, located in the mitochondrial wall, inhibits the release of cytochrome $c$ (58-60). Cytochrome $c$ initiates the process of apoptosis by activating the caspase cascade (59). Interactions between the pro- and anti-apoptotic $\mathrm{Bcl}-2$ family proteins on the outer mitochondrial membrane play a key role in cell survival. Therefore, the Bcl-2/Bax protein ratio may determine the level of cell apoptosis or survival following apoptotic injury (61). In the present study, NRIPoC significantly decreased both Bax and $\mathrm{Bcl}-2$ protein expression and increased the $\mathrm{Bcl}-2 / \mathrm{Bax}$ ratio. These results suggest that the anti-apoptotic effects of NRIPoC may be closely associated with changes in Bcl-2 and Bax expression. The mechanisms through which NRIPoC modulates Bcl-2 and Bax expression following cerebral IRI in vivo are currently unknown. The JAK/STAT signaling pathway transduces stress-activating extracellular chemical signals into cellular responses for a number of pathophysiological processes, such as immunity, inflammation and apoptosis (62). p-STAT1 and p-STAT3 expression have been shown to be increased following focal cerebral ischemia, and the activation of STAT1 may lead to ischemic neuronal necrosis $(42,43,63)$; however, the effects of activated STAT3 following focal cerebral ischemia have not yet been elucidated. The function of activated STAT3 is controversial; some studies have associated it with survival $(43,64)$, while others have related it to cell death (65).

There is no existing evidence that STAT3 is involved in neuronal apoptosis by the regulation of $\mathrm{Bcl}-2$ and Bax expression following cerebral ischemia. Previous studies have confirmed 

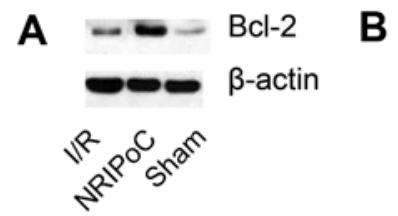

C
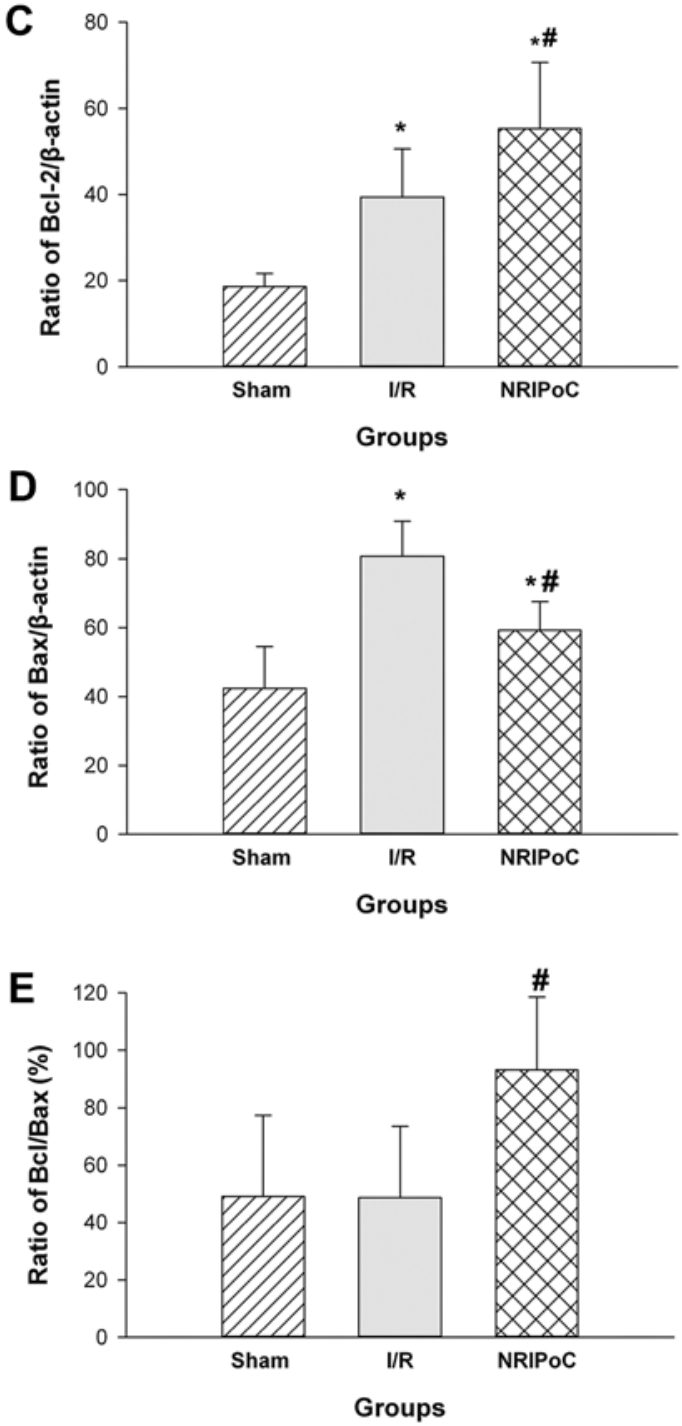

Figure 6. Effects of non-invasive remote ischemic postconditioning (NRIPoC) on ischemia reperfusion injury (IRI)-induced $\mathrm{Bcl}-2$ and $\mathrm{Bax}$ protein expression in ischemic penumbra areas $24 \mathrm{~h}$ after reperfusion $(\mathrm{n}=5)$. (A) $\mathrm{Bcl}-2$ and $(\mathrm{B}) \mathrm{Bax}$ protein expression determined by western blot analysis. (C) Ratio of Bcl-2/ $\beta$ actin; (D) ratio of $\mathrm{Bax} / \beta$-actin; (E) ratio of Bcl-2/Bax. Data are presented as the means \pm standard deviation (SD). ${ }^{*} \mathrm{P}<0.05$ compared with the sham-operated (sham) group; ${ }^{\text {}} \mathrm{P}<0.05$ compared with the ischemia/reperfusion (I/R) group.

that STAT3 alterations affect Bcl-2 and Bax protein expression (decreased Bcl-2 and increased Bax) and induce inflammation and apoptosis in many types of tumor cells (66-68). Lee et al (69) demonstrated that co-activated NF- $\mathrm{kB}$ and STAT3 modulate $\mathrm{Bax} / \mathrm{Bcl}-\mathrm{xL}$ expression and promote cell survival in head and neck squamous cell carcinoma.

In mycosis fungoides tumor cells, some apoptosis-related genes, such as Bcl-2 and Bax, have been identified as STAT3 target genes (66). In primary cortical neurons and murine models of stroke, the activation of the Jak2/Stat 3 pathway by secretoneurin has been found to exert neuroprotective effects
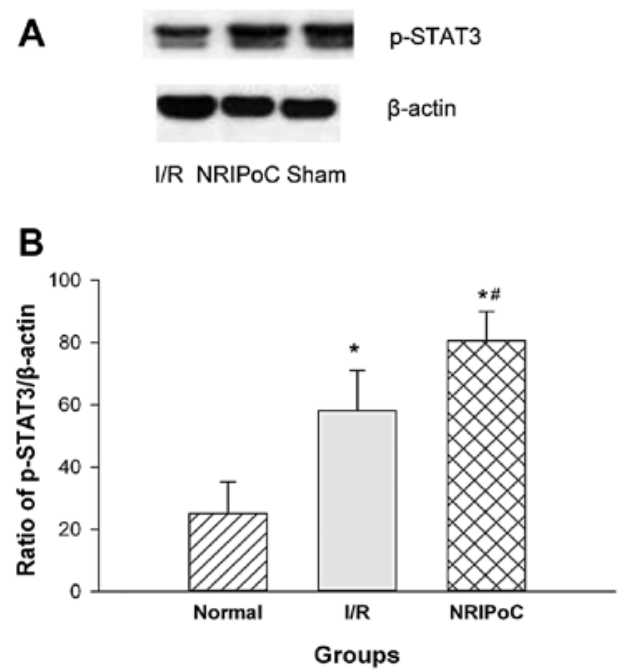

Figure 7. Effects of non-invasive remote ischemic postconditioning (NRIPoC) on ischemia reperfusion injury (IRI)-induced p-STAT3 protein expression in ischemic penumbra areas $24 \mathrm{~h}$ after reperfusion $(\mathrm{n}=5)$. (A) $\mathrm{p}-\mathrm{STAT} 3$ protein expression determined by western blot analysis. (B) Ratio of p-STAT3$\beta$-actin. Data are presented as the means \pm standard deviation (SD) $(n=5)$. "P $<0.05$ compared with the sham-operated (normal) group; ${ }^{\#} \mathrm{P}<0.05$ compared with the ischemia/reperfusion (I/R) group.
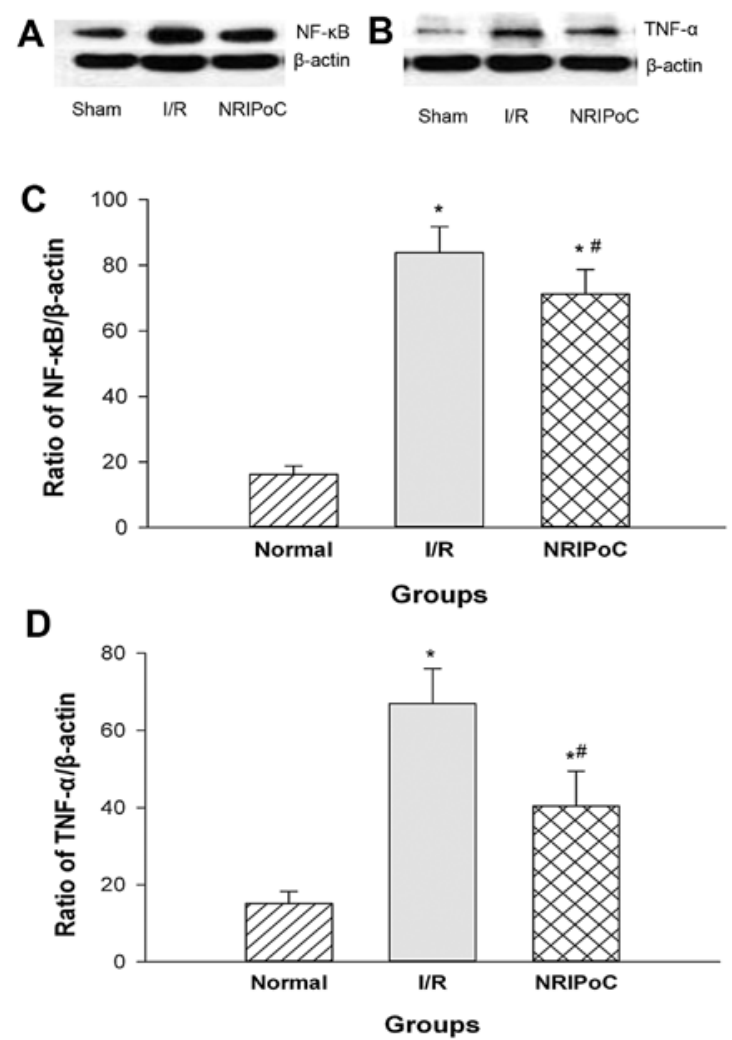

Figure 8. Effects of non-invasive remote ischemic postconditioning (NRIPoC) on ischemia reperfusion injury (IRI)-induced nuclear factor- $\mathrm{\kappa B}(\mathrm{NF}-\mathrm{\kappa} B$ ) and tumor necrosis factor- $\alpha$ (TNF- $\alpha$ ) protein expression determined by western blot analysis in the ischemic penumbra $24 \mathrm{~h}$ after reperfusion (n=5). (A) NF- $\mathrm{kB}$ protein expression; (B) TNF- $\alpha$ protein expression. (C) Ratio of NF- $\mathrm{kB} / \beta$-actin; (D) Ratio of TNF- $\alpha / \beta$-actin. Data are presented as the means \pm standard deviation (SD). ${ }^{*} \mathrm{P}<0.05$ compared with the sham-operated (normal) group; ${ }^{~} \mathrm{P}<0.05$ compared with the ischemia/reperfusion (I/R) group.

and induce neuronal plasticity after hypoxia and ischemic insult (70). Using a mouse model of transient focal cerebral 
ischemia, Jung et al (71) demonstrated that interleukin-6 (IL-6) exerted protective effects against cerebral ischemic injury through IL-6R-mediated STAT3 activation and antioxidative signaling. Similar to previous study by Suzuki et al (43), we found that p-STAT3 expression was increased following focal cerebral ischemia. Furthermore, NRIPoC induced a more pronounced increase in p-STAT3 expression in the ischemic penumbra area. This, coupled with the Bcl-2/Bax ratio was consistent with the decrease in the number of apoptotic neurons and the attenuation of cerebral I/R-induced brain injury. This result provides an experimental basis for the clinical application of NRIPoC for reducing the cerebral infarct volume following stroke through the rescue of the remaining surviving neurons in the penumbra area. These results indicate that p-STAT3 may be an endogenous anti-injury factor, and that the JAK2-STAT3 signaling pathway may mediate the neuroprotective effects of RIPoC though the regulation of $\mathrm{Bcl}-2 /$ Bax expression in rat focal cerebral ischemia. This assumption requires further confirmation by the use of specific inhibitors of the JAK2-STAT3 signaling pathway in order to clarify the effects of STAT3.

The pathogenesis of cerebral IRI involves complex pathophysiological events, such as the excessive production of reactive oxygen species, the excessive activation of glutamate receptors, the overload of intracellular calcium and inflammation $(26,27,72)$. Early inflammatory mediators following cerebral IR are the basis of ischemic injury that then transforms to inflammatory injury. Accumulating evidence suggests that the neuroinflammatory processes occurring after cerebral ischemia involve various pathways and molecules (26). Of these, TNF- $\alpha$ is responsible for the degree of inflammation. $\mathrm{NF}-\kappa \mathrm{B}$ is a critical transcription factor for the maximal expression of several cytokines that are involved in inflammation (73). Whereas TNF- $\alpha$ and IL- $1 \beta$ appear to exacerbate cerebral injury, transforming growth factor- $\beta$ and IL-10 may exert neuroprotective effects during cerebral ischemia reperfusion (26). Activated NF- $\kappa B$ upregulates the transcription of TNF- $\alpha$ and IL-1 $\beta$. An increase in TNF- $\alpha$ activates NF- $\kappa B$, and IL-10 inhibits the activation of NF- $\mathrm{kB}$ mediated by endotoxins. The synthesis of inflammatory mediators is greatly increased by the overexpression of TNF- $\alpha$ following I/R, which results in an imbalance between inflammatory and anti-inflammatory factors and the amplification of the inflammatory cascade. The overexpression of TNF- $\alpha$ may aggravate brain damage following cerebral $I / R$, which is though to be mediated by the transcriptional regulation of inflammatory factors by NF- $\kappa B(26,74)$. The present study demonstrated that TNF- $\alpha$ and NF- $\kappa \mathrm{B}$ protein expression in the ischemic penumbra area was significantly increased following $\mathrm{I} / \mathrm{R}$, and this increase was reduced by NRIPoC, which suggests that the neuroprotective effects of NRIPoC may correlate with the increased inflammatory response in the ischemic penumbra area following IRI. The binding of TNF- $\alpha$ to its receptor, TNF-RI (p55TNFR), induces the caspase cascade, which promotes apoptosis. Several alternative mechanisms of TNF- $\alpha$-induced apoptosis have also been described (75). The downregulation of TNF- $\alpha$ by NRIPoC in the ischemic penumbra area may be one of the reasons why NRIPoC reduced neuronal apoptosis following cerebral I/R. Further studies are required to examine the role of STAT3 signaling in regulating TNF- $\alpha$ and NF- $\mathrm{KB}$ expres- sion induced by cerebral I/R, as well as the neuroprotective effects of NRIPoC.

In conclusion, the present study demonstrates that NRIPoC attenuates cerebral IRI in an in vivo rat model through the alleviation of inflammation, regulation of the STAT3 signaling pathway and Bcl-2 and Bax expression levels, and that these changes are possibly due to the reduction of apoptosis.

\section{References}

1. Bonita R: Epidemiology of stroke. Lancet 339: 342-344, 1992.

2. Murray CJ and Lopez AD: Mortality by cause for eight regions of the world: Global Burden of Disease Study. Lancet 349: 1269-1276, 1997.

3. Gourdin MJ, Bree B and De Kock M: The impact of ischaemiareperfusion on the blood vessel. Eur J Anaesthesiol 26: 537-547, 2009.

4. Murry CE, Jennings RB and Reimer KA: Preconditioning with ischemia: a delay of lethal cell injury in ischemic myocardium. Circulation 74: 1124-1136, 1986.

5. Kitagawa K, Matsumoto M, Tagaya M, et al: 'Ischemic tolerance' phenomenon found in the brain. Brain Res 528: 21-24, 1990.

6. Zhao H, Sapolsky RM and Steinberg GK: Interrupting reperfusion as a stroke therapy: ischemic postconditioning reduces infarct size after focal ischemia in rats. J Cereb Blood Flow Metab 26: 1114-1121, 2006.

7. Wang JY, Shen J, Gao Q, et al: Ischemic postconditioning protects against global cerebral ischemia/reperfusion-induced injury in rats. Stroke 39: 983-990, 2008.

8. Liu X, Chen H, Zhan B, et al: Attenuation of reperfusion injury by renal ischemic postconditioning: the role of NO. Biochem Biophys Res Commun 359: 628-634, 2007.

9. Yellon DM and Dana A: The preconditioning phenomenon: A tool for the scientist or a clinical reality? Circ Res 87: 543-550, 2000.

10. Li CM,Zhang XH, Ma XJ and Luo M: Limb ischemic postconditioning protects myocardium from ischemia-reperfusion injury. Scand Cardiovasc J 40: 312-317, 2006

11. Hong DM, Jeon Y, Lee CS, et al: Effects of remote ischemic preconditioning with postconditioning in patients undergoing off-pump coronary artery bypass surgery - randomized controlled trial. Circ J 76: 884-890, 2012.

12. Vinten-Johansen $\mathrm{J}$ and Shi W: The science and clinical translation of remote postconditioning. J Cardiovasc Med (Hagerstown) 14: 206-213, 2013.

13. Ren C, Yan Z, Wei D, Gao X, Chen X and Zhao H: Limb remote ischemic postconditioning protects against focal ischemia in rats. Brain Res 1288: 88-94, 2009.

14. Sun J, Tong L, Luan Q, et al: Protective effect of delayed remote limb ischemic postconditioning: role of mitochondrial K(ATP) channels in a rat model of focal cerebral ischemic reperfusion injury. J Cereb Blood Flow Metab 32: 851-859, 2012.

15. Wang Q, Zhang X, Ding Q, et al: Limb remote postconditioning alleviates cerebral reperfusion injury through reactive oxygen species-mediated inhibition of delta protein kinase $\mathrm{C}$ in rats. Anesth Analg 113: 1180-1187, 2011.

16. Zhou Y, Fathali N, Lekic T, et al: Remote limb ischemic postconditioning protects against neonatal hypoxic-ischemic brain injury in rat pups by the opioid receptor/Akt pathway. Stroke 42: 439-444, 2011.

17. Ren C, Gao X, Steinberg GK and Zhao H: Limb remote-preconditioning protects against focal ischemia in rats and contradicts the dogma of therapeutic time windows for preconditioning. Neuroscience 151: 1099-1103, 2008.

18. Qi ZF, Luo YM, Liu XR, et al: AKT/GSK3beta-dependent autophagy contributes to the neuroprotection of limb remote ischemic postconditioning in the transient cerebral ischemic rat model. CNS Neurosci Ther 18: 965-973, 2012.

19. Peng B, Guo QL, He ZJ, et al: Remote ischemic postconditioning protects the brain from global cerebral ischemia/reperfusion injury by up-regulating endothelial nitric oxide synthase through the PI3K/Akt pathway. Brain Res 1445: 92-102, 2012.

20. Koch S, Katsnelson M, Dong C and Perez-Pinzon M: Remote ischemic limb preconditioning after subarachnoid hemorrhage: a phase Ib study of safety and feasibility. Stroke 42: 1387-1391, 2011. 
21. Walsh SR, Nouraei SA, Tang TY, Sadat U, Carpenter RH and Gaunt ME: Remote ischemic preconditioning for cerebral and cardiac protection during carotid endarterectomy: results from a pilot randomized clinical trial. Vasc Endovascular Surg 44: 434-439, 2010

22. Hu S, Dong H, Zhang $\mathrm{H}$, et al: Noninvasive limb remote ischemic preconditioning contributes neuroprotective effects via activation of adenosine A1 receptor and redox status after transient focal cerebral ischemia in rats. Brain Res 1459: 81-90, 2012.

23. Zhong H, Gao Z, Chen M, et al: Cardioprotective effect of remote ischemic postconditioning on children undergoing cardiac surgery: a randomized controlled trial. Paediatr Anaesth 23: 726-733, 2013

24. Liu X, Sha O and Cho EY: Remote ischemic postconditioning promotes the survival of retinal ganglion cells after optic nerve injury. J Mol Neurosci 51: 639-646, 2013.

25. Dave KR, Saul I, Prado R, Busto R and Perez-Pinzon MA: Remote organ ischemic preconditioning protect brain from ischemic damage following asphyxial cardiac arrest. Neurosci Lett 404: 170-175, 2006.

26. Lakhan SE, Kirchgessner A and Hofer M: Inflammatory mechanisms in ischemic stroke: therapeutic approaches. J Trans Med 7: 97, 2009.

27. Broughton BR, Reutens DC and Sobey CG: Apoptotic mechanisms after cerebral ischemia. Stroke 40: e331-e339, 2009.

28. MacManus JP and Linnik MD: Gene expression induced by cerebral ischemia: an apoptotic perspective. J Cereb Blood Flow Metab 17: 815-832, 1997.

29. Li Y, Powers C, Jiang N and Chopp M: Intact, injured, necrotic and apoptotic cells after focal cerebral ischemia in the rat. $J$ Neurol Sci 156: 119-132, 1998

30. Feuerstein GZ, Wang X and Barone FC: Inflammatory gene expression in cerebral ischemia and trauma. Potential new therapeutic targets. Ann NY Acad Sci 825: 179-193, 1997.

31. Benjelloun N, Renolleau S, Represa A, Ben-Ari Y and CharriautMarlangue C: Inflammatory responses in the cerebral cortex after ischemia in the P7 neonatal Rat. Stroke 30: 1916-1924, 1999

32. Zeng L, Wang Y, Liu J, et al: Pro-inflammatory cytokine network in peripheral inflammation response to cerebral ischemia Neurosci Lett 548: 4-9, 2013

33. Saito K, Suyama K, Nishida K, Sei Y and Basile AS: Early increases in TNF-alpha, IL-6 and IL-1 beta levels following transient cerebra ischemia in gerbil brain. Neurosci Lett 206: 149-152, 1996.

34. Maddahi A, Kruse LS, Chen QW and Edvinsson L: The role of tumor necrosis factor- $\alpha$ and TNF- $\alpha$ receptors in cerebral arteries following cerebral ischemia in rat. J Neuroinflammation 8: 107, 2011.

35. Vakili A, Mojarrad S, Akhavan MM and Rashidy-Pour A: Pentoxifylline attenuates TNF- $\alpha$ protein levels and brain edema following temporary focal cerebral ischemia in rats. Brain Res 1377: 119-125, 2011

36. Latanich CA and Toledo-Pereyra LH: Searching for NF-kappaB-based treatments of ischemia reperfusion injury. J Invest Surg 22: 301-315, 2009.

37. Ridder DA and Schwaninger M: NF-kappaB signaling in cerebral ischemia. Neuroscience 158: 995-1006, 2009.

38. MacDonald RL and Stoodley M: Pathophysiology of cerebral ischemia. Neurol Med Chir (Tokyo) 38: 1-11, 1998.

39. Zhang S, Qi Y, Xu Y, et al: Protective effect of flavonoid-rich extract from Rosa laevigata Michx on cerebral ischemia-reperfusion injury through suppression of apoptosis and inflammation. Neurochem Int 63: 522-532, 2013.

40. Zhang L, Dong LY, Li YJ, Hong Z and Wei WS: The microRNA miR-181c controls microglia-mediated neuronal apoptosis by suppressing tumor necrosis factor. J Neuroinflammation 9: 211, 2012.

41. Gu JH, Ge JB, Li M, Wu F, Zhang W and Qin ZH: Inhibition of $N F-\kappa B$ activation is associated with anti-inflammatory and antiapoptotic effects of Ginkgolide B in a mouse model of cerebral ischemia/reperfusion injury. Eur J Pharm Sci 47: 652-660, 2012.

42. Justicia C, Gabriel C and Planas AM: Activation of the JAK/STAT pathway following transient focal cerebral ischemia: signaling through Jak1 and Stat3 in astrocytes. Glia 30: 253-270, 2000.

43. Suzuki S, Tanaka K, Nogawa S, Dembo T, Kosakai A and Fukuuchi Y: Phosphorylation of signal transducer and activator of transcription-3 (Stat3) after focal cerebral ischemia in rats. Exp Neurol 170: 63-71, 2001.

44. Satriotomo I, Bowen KK and Vemuganti R: JAK2 and STAT3 activation contributes to neuronal damage following transient focal cerebral ischemia. J Neurochem 98: 1353-1368, 2006
45. Xie HF, Xu RX, Wei JP, Jiang XD and Liu ZH: P-JAK2 and P-STAT3 protein expression and cell apoptosis following focal cerebral ischemia-reperfusion injury in rats. Nan Fang Yi Ke Da Xue Xue Bao 27: 208-211, 218, 2007 (In Chinese).

46. Longa EZ, Weinstein PR, Carlson S and Cummins R: Reversible middle cerebral artery occlusion without craniectomy in rats. Stroke 20: 84-91, 1989.

47. Wang N, Guo QL, Ye Z, Xia PP, Wang E and Yuan YJ: Preconditioning of intravenous parecoxib attenuates focal cerebral ischemia/reperfusion injury in rats. Chin Med J (Engl) 124: 2004-2008, 2011

48. Wei L, Yu SP, Gottron F, Snider BJ, Zipfel GJ and Choi DW Potassium channel blockers attenuate hypoxia- and ischemiainduced neuronal death in vitro and in vivo. Stroke 34: 1281-1286, 2003.

49. Sambrook J, Fritsch EF and Maniatis T: Molecular Cloning: a Laboratory Manual. 2nd edition. Cold Spring Harbor Laboratory Press, New York, 1989.

50. Astrup J, Siesjö BK and Symon L: Thresholds in cerebral ischemia - the ischemic penumbra. Stroke 12: 723-725, 1981.

51. Paciaroni M, Caso V and Agnelli G: The concept of ischemic penumbra in acute stroke and therapeutic opportunities. Eur Neurol 61: 321-330, 2009

52. Tajiri S, Oyadomari S, Yano S, et al: Ischemia-induced neuronal cell death is mediated by the endoplasmic reticulum stress pathway involving CHOP. Cell Death Differ 11: 403-415, 2004.

53. Hu BR, Martone ME, Jones YZ and Liu CL: Protein aggregation after transient cerebral ischemia. J Neurosci 20: 3191-3199, 2000.

54. Xing B, Chen $\mathrm{H}$, Zhang $\mathrm{M}$, et al: Ischemic postconditioning inhibits apoptosis after focal cerebral ischemia/reperfusion injury in the rat. Stroke 39: 2362-2369, 2008.

55. Liu Y, Sercombe R, Xie D, Liu K and Chen L: Inhibition of caspase-9 activation and apoptosis is involved in ischemic preconditioning-induced neuroprotection in rat brain. Neurol Res 29: 855-861, 2007.

56. Prasad SS, Russell M and Nowakowska M: Neuroprotection induced in vitro by ischemic preconditioning and postconditioning: modulation of apoptosis and PI3K-Akt pathways. J Mol Neurosci 43: 428-442, 2011

57. Xia DY, Li W, Qian HR, Yao S, Liu JG and Qi XK: Ischemia preconditioning is neuroprotective in a rat cerebral ischemic injury model through autophagy activation and apoptosis inhibition. Braz J Med Biol Res 46: 580-588, 2013.

58. Kroemer G: Mitochondrial control of apoptosis: an introduction. Biochem Biophys Res Commun 304: 433-435, 2003.

59. Lim ML, Lum MG, Hansen TM, Roucou X and Nagley P: On the release of cytochrome $c$ from mitochondria during cell death signaling. J. Biomed Sci 9: 488-506, 2002.

60. Kuwana T, Mackey MR, Perkins G, et al: Bid, Bax, and lipids cooperate to form supramolecular openings in the outer mitochondrial membrane. Cell 111: 331-342, 2002.

61. Love S: Apoptosis and brain ischaemia. Prog Neuropsychopharmacol Biol Psychiatry 27: 267-282, 2003.

62. Planas AM, Gorina R and Chamorro A: Signalling pathways mediating inflammatory responses in brain ischaemia. Biochem Soc Trans 34: 1267-1270, 2006.

63. Planas AM, Soriano MA, Berruezo M, et al: Induction of Stat3, a signal transducer and transcription factor, in reactive microglia following transient focal cerebral ischaemia. Eur J Neurosci 8: 2612-2618, 1996

64. Yamaguchi K, Itoh Y, Yokomizo C, et al: Blockade of interleukin-6 signaling enhances hepatic steatosis but improves liver injury in methionine choline-deficient diet-fed mice. Lab Invest 90: 1169-1178, 2010

65. Wen TC, Peng H, Hata R, Desaki J and Sakanaka M: Induction of phosphorylated-Stat 3 following focal cerebral ischemia in mice. Neurosci Lett 303: 153-156, 2001.

66. Nielsen M, Kaestel CG, Eriksen KW, et al: Inhibition of constitutively activated Stat 3 correlates with altered Bcl-2/Bax expression and induction of apoptosis in mycosis fungoides tumor cells. Leukemia 13: 735-738, 1999.

67. Dinasarapu AR, Gupta S, Ram Maurya M, et al: A combined omics study on activated macrophages - enhanced role of STATs in apoptosis, immunity and lipid metabolism. Bioinformatics 29: 2735-2743, 2013

68. Spehlmann ME, Manthey CF, Dann SM, et al: Trp53 deficiency protects against acute intestinal inflammation. J Immunol 191: 837-847, 2013. 
69. Lee TL, Yeh J, Friedman J, et al: A signal network involving coactivated NF-kappaB and STAT3 and altered p53 modulates BAX/BCL-XL expression and promotes cell survival of head and neck squamous cell carcinomas. Int J Cancer 122: 1987-1998, 2008.

70. Shyu WC, Lin SZ, Chiang MF, et al: Secretoneurin promotes neuroprotection and neuronal plasticity via the Jak2/Stat 3 pathway in murine models of stroke. J Clin Invest 118: 133-148, 2008.

71. Jung JE, Kim GS and Chan PH: Neuroprotection by interleukin-6 is mediated by signal transducer and activator of transcription 3 and antioxidative signaling in ischemic stroke. Stroke 42: 3574-3579, 2011

72. Allen CL and Bayraktutan U: Oxidative stress and its role in the pathogenesis of ischaemic stroke. Int J Stroke 4: 461-470, 2009.
73. Blackwell TS and Christman JW: The role of nuclear factorkappa B in cytokine gene regulation. Am J Respir Cell Mol Biol 17: 3-9, 1997.

74. Ginis I, Jaiswal R, Klimanis D, Liu J, Greenspon J and Hallenbeck JM: TNF-alpha-induced tolerance to ischemic injury involves differential control of NF-kappaB transactivation: the role of NF-kappaB association with p300 adaptor. J Cereb Blood Flow Metab 22: 142-152, 2002.

75. Jurewicz A, Matysiak M, Tybor K, Kilianek L, Raine CS and Selmaj K: Tumour necrosis factor-induced death of adult human oligodendrocytes is mediated by apoptosis inducing factor. Brain 128: 2675-2688, 2005. 